\title{
EDITORIAL
}

\section{Why would we care about privacy?}

\author{
Tamara Dinev \\ Florida Atlantic University, Boca Raton, U.S.A. \\ European Journal of Information Systems \\ (2014) 23, 97-102. doi: 10.1057/ejis.20I4.I
}

\section{Introduction}

This editorial is about privacy in the information age, future opportunities for research, and how the EJIS community of editors and scholars are uniquely positioned to make a significant impact on the advancement of its understanding. While there are many physical aspects of privacy, the particular relevance and biggest developments concern information privacy. Societal discourse has come to equate privacy and information privacy and this editorial does so as well.

With the ubiquitous collection, storage, analysis, and sharing of digital data, the explosion of information technology (IT), e-commerce, social networks, and government surveillance, privacy has become a pervasive issue in both the private and public sector. The recent revelations of governments' dragnet surveillance of phone calls and other digital communication data of the citizens of the United States and European nations have produced media storms and heightened the tone of political discourse. Friendly relationships among democratic governments were put to test, sensitive cords were struck in some nations that had experienced pervasive surveillance in their history. IT analysts predicted that technology companies may severely suffer economically if citizens and businesses withdrew their use of cloud data storage, social networks such as Facebook, Twitter, Instagram, or user data-intensive companies such as Google, Microsoft, and Apple. Many analysts in the media warned that, if the privacy needs of the citizens and businesses are not met, their willingness to do business online may suddenly topple irreversibly with dramatic results for the economic development.

And yet, despite this storm of revelations and analyses, there does not seem to be a considerable bottom-up political and societal pressure to change the practices of government surveillance and data collection by the private businesses. The society response as captured in poll after poll, seems to be divided and tepid. This may lead politicians and policy makers to think that people have accepted the government explanation of this massive surveillance as the necessary tool to strengthen the security of the nations and thwart terrorist attacks. As in e-commerce, we yet again see the wellknown paradoxical phenomenon: poll after poll registers that people are highly concerned about their privacy. Nevertheless, they freely submit personal information and accept being monitored, both by businesses and government. Thus, feeling uninhibited, businesses and government expand even more their collection and use of personal data and behavior.

There can only be a few explanations for this interesting paradoxical phenomenon: (1) people think they care and are concerned about privacy but actually they are not; (2) people, organizations, and governments do not understand exactly what privacy is and how the lack of it can affect the individual; (3) people understand and want their privacy but do not exactly know the extent and the mechanisms of data collection and what the implications for their privacy are. While we do not know exactly which of the three explanations is adequate, either of them and good research 
evidence (e.g., Mason, 1986; Dhillon et al, 2007; Dinev et al, 2008; Li \& Unger, 2012; Miltgen \& Peyrat-Guillard, 2014) show that societies are still actively searching for the balance between preserving the right amount of privacy (that people will demand and not compromise over), and providing convenience (personalized services and goods in the case of businesses) as well as security (in the case of government surveillance).

In either cases, MIS research can contribute in the most substantial and meaningful way to the societal argument. Dissemination of our findings and arguments are needed more than ever so the MIS scholars can actively participate in the political discourse and meaningfully help shape policies and regulations across countries and within the unions of states. Currently, it seems that in the legislative, regulatory, and policy making circles, the larger part of the scholarly voice about privacy issues is conferred to the legal and social sciences scholars. The MIS voice is not as strongly and convincingly heard, gently reminding us of the old debate about relevance. The MIS field's core mission is to provide a comprehensive treatment of IT within the individual, organizational, and social contexts. In the case of privacy, MIS scholars are called upon again to build a wholesome and convincing 'so what' story about privacy that will be clearly heard and considered. For that to happen, we believe, MIS research needs to reach out and integrate the body of knowledge that has so far been largely ignored because it seemed to go beyond the MIS paradigms established in the last decades. Examples of such body of knowledge are anthropology, culture, regulation, political science, international and public policy, behavioral economics, psychological research on visceral reactions and influences on behavior, and others.

MIS scholars have rightfully so taken big interest and forged a modern, scientific development of the privacy concept. They employed positivist approaches that defined and measured privacy-related constructs and put these constructs in nomological models that sought to bring clarity and understanding of this vague and murky concept. Top MIS journals such as MIS Quarterly, Information Systems Research, European Journal of Information Systems, Journal of Strategic Information Systems, and others have taken to task and published an impressive body of research on privacy (see for references Bélanger \& Crossler, 2011, Li, 2011, Pavlou, 2011, Smith et al, 2011). This by itself is an enormous contribution to the literature and understanding of privacy. MIS curiosity has been heightened by questions such as what is the impact on privacy of commercial and government implementation of emerging technologies such as RFID, GPS, context- and location-aware applications, social networks, mobile devices, etc. (Dhillon et al, 2007; Keith et al, 2013) and how can privacy be still protected while drawing the benefits from these technologies or from any personalization technology for that matter. MIS scholars have been eager to extract the right balance between security and privacy when it comes to government surveillance (Mason, 1986; Kling, 1996; Lyon, 2001; Dinev et al, 2006).
Recently, three comprehensive privacy literature review articles were published (Bélanger \& Crossler, 2011, Li, 2011, Smith et al, 2011) that sought to evaluate the current state of privacy conceptualization in IS and other disciplines and guide the future research in the IS literature. This editorial can hardly match the comprehensiveness of these theory review papers and does not intend to repeat their conclusions. Rather, it attempts to go beyond them as well as to crystalize some unique opportunities for EJIS scholars and editors.

\section{EIS and privacy research}

Why is EJIS uniquely positioned to raise a number of questions and research them in a number of ways to advance the privacy research? First and foremost - the diversity of the community: readers, authors, reviewers, and editors, as astutely noted by Te'eni (2012). EJIS draws from such a diverse body of scholars with so diverse background, training, and interests, that given the diversity of privacy itself, it is a gold mine for privacy research. Immersed in this diversity, the EJIS editorial team actively encourages diversity of genres (Rowe, 2011 and 2012), research methods, cultural lenses, and norms (Rowe, 2011; Baskerville, 2012; Te'eni, 2012) that truly reflect the multicultural nature of Europe while not being limited to it. As Baskerville (2012) noted, it is not the mere acceptance of diversity as it is the passionate pursuit of it that allows diversity to become truly ingrained in the EJIS community and publications. There can hardly be found another concept that is as multidisciplinary and diverse as privacy. As such, it requires diversity - diverse scholars, treatments, views, angles, and genres, including design science (Oetzel and Spiekermann, 2014). It requires active collaboration and integration with other fields, genuine, inimitable understanding of cultures and regulations. Finally, the regulatory and cultural diversity (Miltgen \& Peyrat-Guillard, 2014) of the independent nations in Europe is a platform that EJIS scholars are immersed in, which is an advantage and an opportunity.

\section{Future themes and opportunities for privacy research}

There are several exciting emerging opportunities about privacy research that will broaden our views and have larger impact on the body of knowledge. Some of them are discussed below.

\section{Definition and conceptualization of privacy}

Pavlou (2011) has noted that despite the seemingly simple and short definitions offered for privacy and the tremendous amount of research across disciplines over the years, ambiguity and disagreement still surrounds the concept. The many perspectives, including law, economics, psychology, management, marketing, political and social sciences wait to be incorporated. There is one common thread in all the various aspects and lenses however, and it is the outcome - do the individual, the group, or the 
society feel that their privacy needs are met? If not, what can be done about it without compromising the common good and the convenience? For the answers to emerge, an integrated work of a diverse body of scholars and research is needed.

\section{Anthropological and cultural angle of privacy}

In order to understand where we are and where we will go as a society in valuing privacy, we need to know how we came to cherish it. The anthropological findings, as inconclusive as they are, have found one thing for sure patterns of privacy differ widely from one society to another. Anthropologists posit that privacy is a socially created need and that the demand for privacy depends on the level of social development of the society (McLean, 1995; Cate, 1997). Jaeger (1986) points to the notion of privacy in the Ancient Greece where the activities associated at home were distinguished from the activities in the polis, the city state. Westin (2003) comments that even highly communal and tribal societies have elaborate rituals for maintaining personal distance and solitude. In contrast, Cranston (1973) states that no word can be found in the ancient or medieval cultures that corresponds to the contemporary Western 'privacy', this view is supported by researchers who report that the idea of privacy is not necessarily a part of the human consciousness (Velecky, 1978). The need to privacy has evolved as protection against disclosure of damaging mistakes, bruising, and disapproved contacts (Westin, 1967). Thus, anthropologists conclude, privacy has probably emerged in social systems with well-developed individual competitiveness, strong religious mores, norms, and judgments about what's wrong and what's right. Along with that, a wellformed social and economic elite is present that asserts its need for separateness from the others (Hickson, 1987). Since privacy was practiced predominantly by the privileged class in the last four centuries, the connotation of 'private' has gradually acquired a favorable meaning - the sense of privilege and exclusivity - unlike other types of alienation and separateness that are perceived as deprivation (Hickson, 1987). The increased physical distance between homes rendered the physical privacy a characteristic of everyday life (Hickson, 1987). Although the following industrialization and urbanization have shrunk the physical space between the people, the spread of prosperity and wealth caused more aspirations to privacy by more people, including the middle class (McLean, 1995).

Many questions arise regarding the future of privacy as norms, acceptance of diversity, importance of social status, and mobility change with the explosive evolution of IT. MIS scholars can explore this important lens. Understanding the anthropological and cultural origins of privacy will also help understand the various regulatory approaches and needs across nations regarding privacy. In this respect, EIIS leads the way among the top journals with its history of publishing cross-cultural aspects of privacy (e.g. Dinev et al, 2006; Miltgen \& Peyrat-Guillard, 2014). For example,
Miltgen \& Peyrat-Guillard (2014) showed cultural and generational divides among European citizens in their decision to disclose and protect their personal data. Assumingly, the origins of these differences are rooted in the anthropological development of the European societies. More studies of that nature are definitely needed and EJIS is the perfect home for them.

\section{Regulation}

Historically, the need for definition and conceptualization of privacy originated in the law and the needs of courts to define the boundaries between public and private. When it was only concerning physical privacy, the approach was relatively easy: the boundaries of the physical space such as room, house, private property, satisfied the quest for that definition. The landscape changed when the courts and states found the need to include information and data gathering in the definition of privacy and with this to rigorously redefine public-private boundaries. It is important to remember that it will be the courts and states again who will need the definitions and conceptualization that the scientists will flesh out from their research on privacy. And this is where the largest societal impact of MIS privacy research lies.

Understanding the legal landscape is of utmost importance since in the age of globalization and global data sharing and trans border data flow, the various national regulations can pose significant problems. With the emergence of global data standards and interorganizational data exchanges, regulation will play a big role in what is considered private data. That will influence the exchange of common data, data synchronization, and interorganizational systems performance (de Corbière \& Rowe, 2013). In certain jurisdictions, including many European countries, privacy is viewed as a fundamental right enshrined in many international and national constitutions. Article 8 of the European Convention of Human Rights provides a right to 'respect for privacy and family life'. But it may be very different in other countries and unions.

\section{Privacy and convenience. Privacy paradox}

Mentioned briefly above in the case of government surveillance, one of the most fascinating elements in privacy research is what economists call 'privacy paradox'. This is the phenomenon where individuals act contrary to their stated preferences, that is, they express high privacy concerns but behave in contradictory ways to these concerns by giving personal and sensitive information possibly in exchange for some convenience and benefits (Pavlou, 2011). This widely known phenomenon prompted economists to define privacy as a commodity (not a human right, not a value) that can be traded if enough incentives such as convenience or monetary gains/savings are given. This view prompted MIS researchers to focus on developing models of rational cost-benefit analysis, or what came to be known as 'privacy calculus' - an act of rational consumers who reveal private information in 
exchange of expected benefits such as convenience or discounts (Culnan \& Armstrong, 1999, Pavlou, 2011, Smith et al, 2011, Li \& Unger, 2012, Keith et al, 2013).

It is now believed that there is more in the privacy paradox than the perfectly rational pictures we are used to build. In a recent editorial, Goes (2013) calls for incorporating the research of behavioral economics into MIS, particularly when it comes to privacy and security. He refers to the findings of Lowenstein (1996), the Nobel laureate Kahneman (Kahneman et al, 1982, 1991) and other notable behavioral economists and psychologists who taught us that our brain is actually not perfectly rational. It has many biases that play role in our decision making. We cannot avoid these biases since they are evolutionary, nor are we automatically aware of them. Acquisti \& Grossklags (2005) refer to this set of systematic psychological deviations from rationality as bounded rationality and suggest that the assumption of perfect rationality might not adequately capture all cases and nuances of an individual's privacy behavior. Experiments conducted by Acquisti and his colleagues (Acquisti, 2004; Acquisti \& Grossklags, 2005; Grossklags \& Acquisti, 2007; Tsai et al, 2011; Acquisti et al, 2012) and by Li et al (2011) have been designed to capture contextual or situational effects on privacy behaviors and have used the principles of bounded rationality from behavioral economics to explain the privacy paradox. Framing, anchoring, loss aversion, endowment are some of the cognitive biases that have been identified that heavily influence the individual's preferences. Probably, some of these biases play role in the paradoxical, context-dependent privacy behaviors.

Another emerging stream of research on privacy that may help explaining the privacy paradox comes from the psychological view of the way the brain processes information and comes to making a decision, the so-called Elaboration Likelihood Model (ELM; Petty \& Cacioppo, 1986; Petty \& Wegener, 1998). The rational, cost-benefit models developed by the MIS researchers actually assume what the ELM refers to as Central Route Processing: responses to external stimuli yield deliberate analyses, which lead to fully informed privacy-related behaviors that are directed by rationally formed attitudes. Individuals reflect thoughtfully and deliberately on their behaviors involving privacy options. However, there is another route of processing information from external stimuli, and that is the Peripheral Route. This is the route of low-effort thinking and heuristic processes when shaping one's behavior. Rational models overlook the peripheral processing route. When following the peripheral route, attitudes form, change, and direct behavior without extensive information processing and deliberate analysis. Instead, information processing and decisions are enacted by simple, relatively automatic cognitive heuristics processes that are derived from past experiences and associations. It has been shown that affect, emotional state, moods, and other factors tend to have an impact on which route a decision making will be processed through. Recently Zhang (2013) has published an important research on the role of affect. Following the works of Lowenstein (1996) on visceral influences on decision making, Dinev et al (2013) have extracted ELM-inspired propositions that aim at explaining many privacy behaviors that fall outside the bounds of the previously proposed privacy completely rational macro-models (see also the work of Lowry et al (2012)). Needless to say, research in this area is just beginning.

We are grateful to Frantz Rowe for the opportunity for this editorial and for his helpful guidance. Appreciation and gratitude are also extended to $\mathrm{H}$. Jeff Smith, Bjoern Niehaves, and Paul Benjamin Lowry for their helpful suggestions and comments.

\section{In this issue of EJIS}

The first paper in this issue, 'Cultural and generational influences on privacy concerns: a qualitative study in seven European countries' co-authored by Caroline L. Miltgen and Dominique Peyrat-Guillard from Angers University, presents an extensive qualitative multicultural study of privacy concerns across seven European countries from four distinct blocks. The study has a significant and unique contribution to the privacy literature. It is one of the very few that offers qualitative perspective by analyzing the words of the European citizens themselves. It is truly multi-cultural involving seven countries with truly diverse background as opposed to the typical studies that involve two or three nations. This allows data mapping and analysis at depths that are not possible when fewer countries are studied. Clear distinction is found across age and across geographical axes (East-West and South-North) regarding responsibility, trust, attitudes towards regulation, and risks of data disclosure. As such, this study is very important to inform regulation and policies in the European Union regarding data disclosure.

The second paper, 'A systematic methodology for privacy impact assessments: a design science approach' by Marie C. Oetzel and Sarah Spiekermann from Vienna University of Economics and Business, offers a methodology for institutions to systematically consider privacy issues by using a step-by-step privacy impact assessment (PIA). The study follows the design science research paradigm and offers a useful approach that helps practitioners understand the relevant privacy regulation landscape and framework. The method is tested and adopted by the German Federal Office for Information Security. A PIA seven-step process is triggered when a new system is implemented or an existing one is upgraded. During the process, existing documentation and resolutions are updated and checked for validity. In addition to describing the seven steps of the process, the authors provide key findings of the exemplary PIA for a retail scenario. These key findings influenced the design of RFID applications in the retail sector and for related business processes. The PIA methodology enables practitioners to integrate the privacy-by-design concept into their system development life cycle. 
The third article 'A process model for analyzing and managing flexibility in information systems', co-authored by Ram L. Kumar and Antonis C. Stylianou from University of North Carolina, aims for a better understanding and management of different types of flexibility in order to enhance organizational performance. The paper starts by presenting a summary on the relevant literature related to IS flexibility. The paper then proceeds using a grounded theory approach to map the identified IS flexibility aspects into research contributions and gaps. Data collected from cases in the academic and professional publications is then analyzed to identify flexibility context factors, why flexibility is needed, as well as detecting synergies and tradeoffs between different flexibility aspects. The authors deploy a process model for the analyses and management of flexibility that takes into account a broad range of IS-related management decisions and does not confine itself to decisions regarding IS infrastructure's flexibility. They identify different types of flexibilities and explain why they are important. They closely examine relationships and tradeoffs between them, and recognize strategies and moderating factors for proactively managing flexibility. The paper successfully manages to catalog major flexibility contextual factors, as well as reasons why flexibility is needed. Finally, the authors are able to establish three main IS flexibility categories (flexibility in IS operations, flexibility in IS systems and services development and deployment, and flexibility in IS management) that encompass 10 IS flexibility types.

The fourth research article is conducted by Michelle Carter from University of Nebraska Omaha, Ryan Wright from University of Massachusetts Amherst, Jason Bennett Thatcher and Richard Klein from Clemson University and is entitled 'Understanding online customers' ties to merchants: the moderating influence of trust on the relationship between switching costs and e-loyalty'. Over a sample of 299 online travel users, the study tests the relative influence of trust compared to switching costs on customers' online loyalty (e-loyalty) with online travel service providers. Switching costs may be financial, procedural, or relational. Using structural equation modeling techniques, the authors find that trust is a more important predictor for e-loyalty than switching costs. Further, the authors find initial support that trust moderates the relationship between switching costs and e-loyalty. The relative comparison between trust and switching costs and their effects on e-loyalty helps to explain which type of customer commitment - affective or continuance- has greater impact on e- loyalty.

The fifth article 'Using organizational influence processes to overcome IS implementation barriers: lessons from a longitudinal case study of SPI implementation' written by Ojelanki Ngwenyama from Ryerson University and University of Cape Town and Peter Axel Nielsen from Aalborg University and University of Agder, looks at organizational political behaviors as a central feature of influencing IS project implementation. Inside a large software development company, the researchers took up the mission to investigate, over a 23-months time span, the key dimensions, the organizational context, direction, targets, and tactics of influence deployed by the Software Process Improvement (SPI) team to achieve success under conditions that would not normally yield successful results. The software development company had set as its strategic missions to bring up its ITC on the CMM-SW process maturity scale, and increase software productivity. The key focus of this study was how the SPI group designed and implemented a coordinated set of organizational influence processes (OIPs) and was successful. The study elaborates on three critical aspects of the influence strategy's dynamics: (a) structural conditions and the design of influence strategies; (b) power dependencies and choices of direct influence tactics; and (c) choices of influence tactics for enrolling intermediaries, especially when implementation managers are dependent upon organizational actors for the realization of project outcomes. The study concludes with support that OIPs can be effective in overcoming barriers to IS implementation success.

The sixth article, 'Board-level IT governance and organizational performance' presented by Ofir Turel from California State University and Chris Bart from McMaster University, investigates the positive influence that board members exercise over IT governance (ITG) to increase organizational performance. The study is based on empirical data drawn from 171 answers collected from Canadian board members. The authors show that higher levels of ITG exercised by the board of directors lead to better organizational performance, and that the organizations' IT use mode (e.g. high need for fast and reliable IT, or high need for new IT for defensive purposes) has an influence over the ITG efforts exercised by the boards of directors. Therefore, board members choose the level of ITG they exercise based on their organizations' need for IT. Furthermore, the quantitative study implies that while board members employ ITG based on their organizations' IT needs, a high level of ITG increases organizational performance irrespective of whether it is aligned to the level prescribed by an organization's IT mode. This is further analyzed in the qualitative part of the study showing some interesting lessons for IT managers. For theoretical contributions, the paper shows that board-level ITG can be an important IT management capability that is often ignored in MIS research.

The editors warmly thank this issue's associate editors on their investment and contribution to this prominent issue of EJIS: Robert Winter (University of St. Gallen, Switzerland), Tamara Dinev (Florida Atlantic University, U.S.A.), Robin Poston (University of Memphis, U.S.A.), Merrill Warkentin (Mississippi State University, U.S.A.), Mark Lycett (Brunel University, U.K.), Régis Meissonier (Montpellier II University, France). Thanks also go to Myriam Raymond (Université de Nantes) for assembling the summaries for this issue's editorial. 


\section{References}

ACQUISTI A (2004) Privacy in electronic commerce and the economics of immediate gratification. In Proc. ACM Electronic Commerce Conference, pp 21-29, ACM, New York.

ACQUISTI A and GROSSKLAGS I (2005) Privacy and rationality in individual decision making. IEEE Security and Privacy 3(1), 26-33.

ACQUISTI A, JOHN L and LOEWENSTEIN G (2012) The impact of relative standards on the propensity to disclose. Journal of Marketing Research 49(2), 160-174.

BASKERVILLE R (2012) Reviving the IT in the IS. European Journal Information Systems 21(6), 587-591.

BÉLANGER F and CROSSLER RE (2011) Privacy in the digital age: a review of information privacy research in information systems. MIS Quarterly 35(4), 1017-1041.

CATE FH (1997) Privacy in the Information Age. Brookings Institution Press, Washington, D.C.

CRANSTON M (1973) What Are Human Rights? Taplinger Pub Co., New York, NY.

CULNAN MJ and ARMSTRONG PK (1999) Information privacy concerns, procedural fairness and impersonal trust: an empirical investigation. Organization Science 10(1), 104-115.

DE CORBIÈrE F and ROWE F (2013) From ideal data synchronization to hybrid forms of interconnections: architectures, processes, and data. Journal of the Association for Information Systems 14(10), 550-584.

DHILLON G, BACKHOUSE J and RAY A (2007) Editorial: JSIS security and privacy special issue. Journal of Strategic Information Systems 16(2), 127-129.

Dinev T, Bellotto M, Hart P, Russo V, Serra I and Colautti C (2006) Privacy calculus model in e-commerce - a study of Italy and the United States. European Journal of Information Systems 15(4), 389-402.

DINEV T, HART P and MULLEN M (2008) Internet privacy concerns and beliefs about government surveillance - an empirical investigation. Journal of Strategic Information Systems 17(3), 214-233.

DINEV T, SMITH HJ and MCCONNEL A (2013) Privacy macromodels: are we really rational? Work in progress.

GOES P (2013) Information systems research and behavioral economics. Editor's comments. MIS Quarterly 37(3), iii-viii.

GROSSKLAGS J and ACQUISTI A (2007) When 25 cents is too much: an experiment on willingness-to-sell and willingness-to-protect personal information. Proceedings of the 6th Workshop Economics of Information Security, WEIS, Pittsburgh, PA.

HICKSON RF (1987) Privacy in a Public Society: Human Rights in Conflict. Oxford University Press, New York, NY.

JAEGER WW (1986) Paideia: The Ideals of Greek Culture. Oxford University Press, New York, NY.

KAHNEMAN D, KNETSCH KL and THALER RH (1991) Anomalies: the endowment effect, loss aversion, and status quo bias. Journal of Economics Perspectives 5(1), 193-206.

KAHNEMAN D, SLOVIC P and TVERSKY A (1982) Judgment under Uncertainty: Heuristics and Biases. Cambridge University Press, New York, NY.

KeITH M, ThOMPSON SC, HALE J, LOWRY PJ and GREeR C (2013) Information disclosure on mobile devices: re-examining privacy calculus with actual user behavior. International Journal of Human-Computer Studies 71(12), 1163-1173.

KLING R (1996) Information technologies and the shifting balance between privacy and social control. Computerization and Controversy: Value Conflicts and Social Control. (KLING R, Ed), 2nd edn, pp 614-633, Academic Press Inc, San Diego, CA.
LI T and UNGER T (2012) Willing to pay for quality personalization? Tradeoff between quality and privacy. European Journal of Information Systems 21(6), 621-642.

LI Y (2011) Empirical studies on online information privacy concerns: literature review and an integrative framework. Communications of the Association for Information Systems 28(1), Available at: http://aisel .aisnet.org/cais/vol28/iss1/28/.

LI H, SARATHY R and XU H (2011) The role of affect and cognition on online consumers' decision to disclose personal information to unfamiliar online vendors. Decision Support Systems 51(3), 434-445.

LOEWENSTEIN G (1996) Out of control: visceral influences on behavior. Organizational Behavior and Human Decision Processes 65(3), 272-292.

LOWRY PI, MOOdY GD, VANCE A, JENSEN M, JeNKINS JL and WeLLS T (2012) Using an elaboration likelihood approach to better understand the persuasiveness of website privacy assurance cues for online consumers. Journal of the American Society for Information Science and Technology 63(4), 755-766.

LYON D (2001) Surveillance Society: Monitoring Everyday Life. Open University Press, Buckingham, PA.

MASON RO (1986) Four ethical issues of the information age. MIS Quarterly 10(1), 4-12.

MCLEAN D (1995) Privacy and Its Invasion. Praeger, Westport, CT.

Miltgen CL and Peyrat-Guillard D (2014) Cultural and generational influences on privacy concerns: a qualitative study in seven European countries. European Journal of Information Systems 23(2), 97-102.

OETZEL MC and SPIEKERMANN S (2014) A systematic methodology for privacy impact assessments: a design science approach. European Journal of Information Systems 23(2), 97-102.

PAVLOU PA (2011) State of the information privacy literature: where are we now and where should we go? MIS Quarterly 35(4), 977-988.

PETTY RE and CACIOPPO IT (1986) Communication and Persuasion: Central and Peripheral Routes to Attitude Change. Springer-Verlag, New York, NY.

PETTY RE and WEGENER DT (1998) Attitude change: multiple roles for persuasion variables. In Handbook of Social Psychology (GILBERT DT, FISKE ST and LINDZEY G, Eds) Vol. 1, 4th edn, pp 323-390, McGraw-Hill, New York, NY.

ROWE F (2011) Towards a greater diversity in writing styles, argumentative strategies and genre of manuscripts. European Journal of Information Systems 20(5), 491-495.

ROWE F (2012) Toward a richer diversity of genres in information systems research: new categorization and guidelines. European Journal of Information Systems 21(5), 469-478.

SMITH HJ, DINEV T and XU H (2011) Information privacy research: an interdisciplinary review. MIS Quarterly 35(4), 989-1015.

TE'ENI D (2012) What's communication got to do with IT? European Journal of Information Systems 21(4), 341-344.

TSAI JY, EGELMAN S, CRANOR L and ACQUISTI A (2011) The effect of online privacy information on purchasing behavior: an experimental study. Information Systems Research 22(2), 254-268.

VELECKY L (1978) The concept of privacy. In Privacy (YounG J, Ed), pp 13-34, John Wiley and Sons, Chichester, New York, NY.

WESTIN AF (1967) Privacy and Freedom. Atheneum, New York, NY.

WESTIN AF (2003) Social and political dimensions of privacy. Journal of Social Issues 59(2), 431-453.

ZHANG P (2013) The affective response model: a theoretical framework of affective concepts and their relationships in the ICT context. MIS Quarterly 37(1), 247-274. 